\title{
COUPLING BEM AND VEM FOR THE ANALYSIS OF COMPOSITE MATERIALS WITH DAMAGE
}

\author{
MARCO LO CASCIO \\ Department of Engineering, University of Palermo \\ Viale delle Scienze, Edificio 8, 90128 \\ Palermo, Italy \\ marco.locascio01@unipa.it \\ IVANO BENEDETTI \\ Department of Engineering, University of Palermo \\ Viale delle Scienze, Edificio 8, 90128 \\ Palermo, Italy \\ ivano.benedetti@unipa.it \\ Received (Day Month Year) \\ Revised (Day Month Year)
}

\begin{abstract}
Numerical tools able to predict and explain the initiation and propagation of damage at the microscopic level in heterogeneous materials are of high interest for the analysis and design of modern materials. In this contribution, we report the application of a recently developed numerical scheme based on the coupling between the Virtual Element Method (VEM) and the Boundary Element Method (BEM) within the framework of continuum damage mechanics (CDM) to analyze the progressive loss of material integrity in heterogeneous materials with complex microstructures. VEM is a novel numerical technique that, allowing the use of general polygonal mesh elements, assures conspicuous simplification in the data preparation stage of the analysis, notably for computational micro-mechanics problems, whose analysis domain often features elaborate geometries. BEM is a widely adopted and efficient numerical technique that, due to its underlying formulation, allows reducing the problem dimensionality, resulting in substantial simplification of the pre-processing stage and in the decrease of the computational effort without affecting the solution accuracy. The implemented technique has been applied to an artificial microstructure, consisting of the transverse section of a circular shaped stiff inclusion embedded in a softer matrix. BEM is used to model the inclusion that is supposed to behave within the linear elastic range, while VEM is used to model the surrounding matrix material, developing more complex nonlinear behaviors. Numerical results are reported and discussed to validate the proposed method.
\end{abstract}

Keywords: Fibre-reinforced Composite Materials; Computational Micro-mechanics; Computational Homogenization; Virtual Element Method; Boundary Element Method.

\section{Introduction}

Employing accurate and effective computational tools is today an essential item in the analysis and design of novel materials for various structural applications. Ef- 
fective computational tools complement experimental activities, allowing the early selection and preliminary analysis of the most promising solutions among several alternatives, thus speeding up and reducing the cost of the overall development process.

Materials design is often driven by the ability to engineer their microstructure by suitable coupling of different phases whose interaction is optimized to achieve desired properties at the scale of the considered application. Within this framework, computational micro-mechanics plays an important role.

This contribution reports the application of a recently developed numerical technique ${ }^{1,2,3}$ coupling VEM with BEM for the analysis of heterogeneous materials with damage.

VEM ${ }^{4}$ extends the features of FEM to very general polygonal and polyhedral mesh elements, including irregular or non-convex elements as well as elements featuring curved boundaries. For such a reason, VEM provides a powerful, flexible tool for computational micro-mechanics problems, which generally feature phases with complex geometries that may also exhibit non-linear constitutive responses ${ }^{5,6,7}$.

BEM ${ }^{8,9}$ is a numerical technique widely employed for the solution of several classes of problems in solids and materials mechanics, including computational homogenization of materials with complex morphology or constitutive behavior $10,11,12,13,14,15,16,17$. The technique, based on a boundary integral reformulation of the considered problem, allows reducing the analysis dimensionality, e.g. from volumes to surfaces, resulting in substantial simplification of the pre - processing stage and in the decrease of the number of degrees of freedom, without penalties on the solution accuracy.

The content of this contribution is organized as follows: $i$ ) first, a Section providing a brief overview of the employed formulation, with some details about the two-dimensional linear elastic BEM formulation, the lowest-order VEM formulation for two-dimensional domains exhibiting isotropic damage, and some details about the coupling between the two techniques; $i i)$ then a Section reporting an application to a fiber-reinforced composite material developing damage in the matrix phase; iii) lastly, a Section providing some concluding remarks.

\section{Formulation}

The multi-region two-dimensional domain $\Omega \subset \Re^{2}$ with external boundary $\Gamma$, shown in Fig. 1, is considered in this study. It is assumed that no body forces act within $\Omega$, but either displacements or tractions can be enforced on the boundary $\Gamma$. The problem domain $\Omega$ is the union of two sub-domains, namely $\Omega^{\mathrm{B}}$ and $\Omega^{\mathrm{V}}$, which represent, respectively, the transverse section of a fiber and the surrounding polymer matrix in a polymer fiber-reinforced composite. The two sub-domains share the interface $S \equiv \Gamma^{\mathrm{B}}$.

BEM is used to model the fiber inclusion, which is supposed to behave within the linear elastic range, while VEM is used to model the surrounding matrix, developing 
$\begin{array}{lllll}\text { October } 2021 & 12: 11 & \text { WSPC/INSTRUCTION } & \text { FILE }\end{array}$
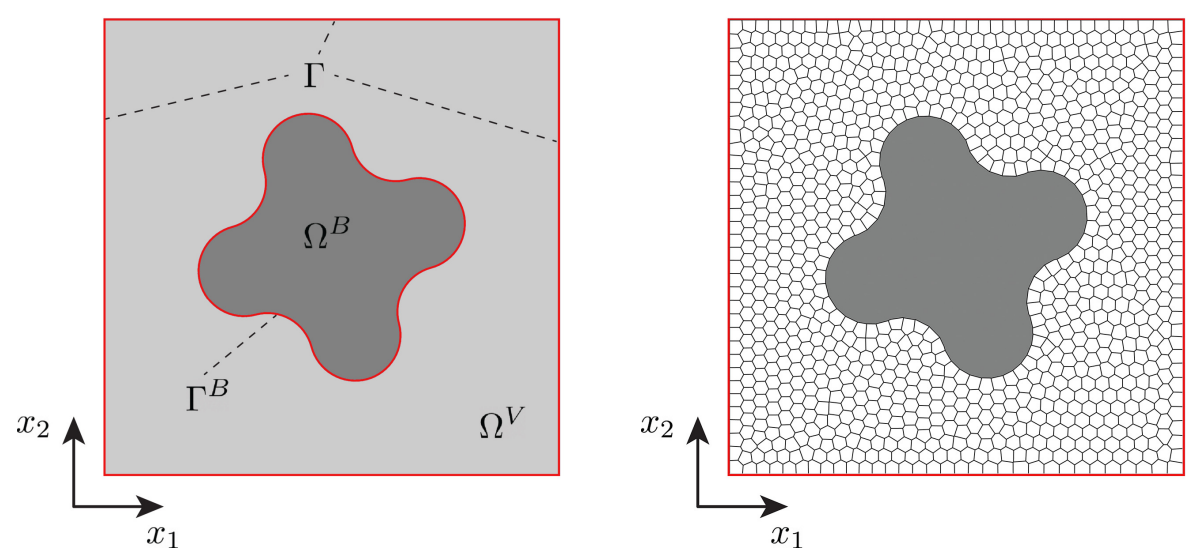

Fig. 1. Sample VEM/BEM analysis domain: geometry (left) and mesh (right).

more complex non-linear behaviours. With respect to standard FEM, VEM offers the advantage of extended flexibility in terms element topology and shapes. The technique ensures convergence and accuracy also for highly distorted meshes and even non-convex elements, which would be considered pathological and problematic in standard FEM analyses. Such a feature relieves the mesh quality requirements for the employed meshes, thus reducing the need of mesh consistency checks in the pre-processing stage of the analysis, which may prove highly beneficial when a large number of morphologies have to be generated and analysed, as often the case in computational micro-mechanics ${ }^{6}$.

For the purpose of the analysis, $\Omega^{\mathrm{V}}$ is partitioned into several polygons of general shape, while the boundary $S \equiv \Gamma^{\mathrm{B}}$ is divided into several straight segments, which form the edges of the polygonal elements in $\Omega^{\mathrm{V}}$ lying in the proximity of the interface between the two sub-domains, see Fig. 1. For further details about the adopted meshing procedures and related convergence analyses, the interested readers are referred to Ref. 3.

\subsection{Boundary Element modeling of the inclusion}

For the sub-domain $\Omega^{\mathrm{B}}$ with smooth boundary $S=\Gamma^{\mathrm{B}}$, if no body forces are applied, the boundary integral equation (BIE) for the displacements components $u_{j}$ at a boundary collocation point $\mathbf{x}_{0} \in S$ can be written, using tensor notation with $i, j=x, y$, as in Ref. 8 .

$$
\frac{1}{2} u_{j}\left(\mathbf{x}_{0}\right)=\int_{S} G_{i j}\left(\mathbf{x}_{0}, \mathbf{x}\right) t_{j}(\mathbf{x}) d s-\int_{S} H_{i j}\left(\mathbf{x}_{0}, \mathbf{x}\right) u_{j}(\mathbf{x}) d s,
$$

where $u_{i}(\mathbf{x})$ and $t_{i}(\mathbf{x})$ are the displacement and traction components at the integration boundary point $\mathbf{x}, G_{i j}\left(\mathbf{x}_{0}, \mathbf{x}\right)$ and $H_{i j}\left(\mathbf{x}_{0}, \mathbf{x}\right)$, respectively, are the components of the two-dimensional elasto-static fundamental solution for displacements and 
$\begin{array}{lllll}\text { October } 21, & 2021 & 12: 11 & \text { WSPC/INSTRUCTION } & \text { FILE }\end{array}$

Revised'Manuscript 'UNMARKED

tractions under plane strain assumptions. The numerical treatment of the boundary integral formulation expressed by Eq. (1) is based on the subdivision of the boundary $S$ into a collection of $m$ straight elements $\Delta S_{k}$ and a convenient approximation of the boundary displacement and traction components in terms of shape functions and nodal values. A linear approximation of displacements and tractions over each boundary element

$$
\mathbf{u}(\xi)=\mathbf{N}(\xi) \mathbf{u}^{k}, \quad \mathbf{t}(\xi)=\mathbf{N}(\xi) \mathbf{t}^{k}
$$

is assumed, where $\mathbf{N}(\xi) \in \mathbb{R}^{2 \times 4}$ is the matrix collecting 1D linear shape functions for the boundary segment $\Delta S_{k}$, expressed as function of the natural coordinate $\xi$, and $\mathbf{u}^{k}, \mathbf{t}^{k} \in \mathbb{R}^{4 \times 1}$ collect, respectively, nodal components of displacements and tractions associated with the boundary segment $\Delta S_{k}$. This approach ensures a straightforward treatment of the interface conditions between the boundary element and the lowest-order VEM elements used to model the contiguous matrix.

Eq. (1) can be numerically integrated using the procedures described, e.g., in Ref. 9. Such numerical integration, repeated for Eq. (1) written for the set of all the collocation points chosen along the boundary $S$, leads to a global system of linear algebraic equations of the form

$$
\mathbf{H} \mathbf{U}^{\mathrm{B}}=\mathbf{G} \mathbf{T}^{\mathrm{B}}
$$

where the vectors $\mathbf{U}^{\mathrm{B}}, \mathbf{T}^{\mathrm{B}} \in \mathbb{R}^{2 m \times 1}$ collect the components of displacements and tractions of all collocation nodes identified along the boundary $S$ and $\mathbf{H}, \mathbf{G} \in$ $\mathbb{R}^{2 m \times 2 m}$ collect the coefficients obtained from the numerical integration of Eq. (1) associated to such collocation points. Since the BEM domain identifies an inclusion in the analyzed domain, both $\mathbf{U}^{\mathrm{B}}$ and $\mathbf{T}^{\mathrm{B}}$ are unknown quantities that must be determined from the analysis.

\subsection{Virtual Element modeling of the matrix}

The lowest-order VEM formulation is employed to model the domain $\Omega^{\mathrm{V}}$, which is here associated with the polymer matrix of a fiber-reinforced composite material.

For a general polygonal element $E$, the element degrees of freedom are the values of the displacements components at each of its $n$ vertex, which are collected into the vector $\mathbf{u}_{E}$. Analogously to the standard FEM, it is assumed that the displacements field $\mathbf{u}$ within the element can be expressed as

$$
\mathbf{u}=\mathbf{N}(\xi, \eta) \mathbf{u}_{E}
$$

where $\mathbf{N}(\xi, \eta)$ is the generic matrix containing the virtual shape functions $N_{v}(\xi, \eta)$ associated with each vertex $v$. Differently from standard FEM however, such shape functions are known only on the element edges of $E$, where they are globally continuous linear polynomials. Since the shape functions $N_{v}(\xi, \eta)$ are not explicitly known within the polygonal element, an explicit expression for the strains is not 
available. An approximated constant strain field $\varepsilon_{\Pi}$ is assumed within each element, which can be computed from the degrees of freedom $\mathbf{u}_{E}$ as

$$
\varepsilon_{\Pi}=\Pi_{E} \mathbf{u}_{E}
$$

where $\Pi_{E} \in \mathbb{R}^{3 \times 2 n}$ is the matrix representation of a projection operator defined as ${ }^{18}$

$$
\boldsymbol{\Pi}_{E}=\frac{1}{A_{E}} \sum_{v=1}^{n} \int_{e_{v}} \mathbf{N}_{v}^{E} \mathbf{N}(\boldsymbol{\eta}) d s, \quad \mathbf{N}_{v}^{E}=\left[\begin{array}{ccc}
n_{x} & 0 & n_{y} \\
0 & n_{y} & n_{x}
\end{array}\right]^{\mathrm{T}}
$$

where $A_{E}$ is the area of the polygonal element $E$, bounded by its $n$ edges $e_{v}$ and $\mathbf{N}_{v}^{E}$ is the matrix containing the components $n_{x}$ and $n_{y}$ of the outward unit normal vector over each edge. The integrals appearing at the right-hand side of Eq. (6) are exactly computable, since the restriction of the virtual shape functions $N_{v}$ to such edges are known piece-wise linear polynomials.

The VEM elemental stiffness matrix $\mathbf{K}_{E}$ is given by the sum of two terms

$$
\mathbf{K}_{E}=\mathbf{K}_{E}^{c}+\mathbf{K}_{E}^{s} \text {. }
$$

The consistency contribution is given by

$$
\mathbf{K}_{E}^{c}=A_{E} \mathbf{\Pi}_{E}^{\mathrm{T}} \mathbf{C}^{0} \mathbf{\Pi}_{E},
$$

where $\mathbf{C}^{0}$ is the material stiffness tensor in Voigt notation. On the other hand, $\mathbf{K}_{E}^{s}$ is a stabilization term that may be computed as described, e.g., in Ref. 4. Its presence is motivated by the need to avoid zero-energy modes not associated with rigid body motion, which may arise as a consequence of the fact that the approximate strains $\varepsilon_{\Pi}$ are assumed constant within the element, while the displacements $\mathbf{u}$ are piece-wise linear on the element edges, which in general may induce incompatibility between $\varepsilon_{\Pi}$ and the nodal degrees of freedom $\mathbf{u}_{E}$. For such a reason, a stabilization strategy similar to that used in the standard FEMs for elements with reduced integration is employed in the VEM, motivating the presence of the stabilization matrix $\mathbf{K}_{E}^{s}$, which ensures the proper rank of the matrix $\mathbf{K}_{E}$.

The equivalent nodal forces $\mathbf{F}_{E}$ are computed as in the standard FEM from specified tractions $\overline{\mathbf{t}}$ over the element boundary $\partial E=\bigcup e_{v}$, i.e.

$$
\mathbf{F}_{E}=\int_{\partial E} \mathbf{N}^{\mathrm{T}}(\xi, \eta) \overline{\mathbf{t}} d s .
$$

Once the elemental stiffness matrices and load vectors are built, the assembly of the VEM global matrices and vectors can be performed following the standard FE procedures, to obtain the following sets of equations for the VEM sub-domain

$$
\mathbf{K}^{\mathrm{V}} \mathbf{U}^{\mathrm{V}}=\mathbf{F}^{\mathrm{V}}
$$

where $\mathbf{K}^{\mathrm{V}}, \mathbf{U}^{\mathrm{V}}$ and $\mathbf{F}^{\mathrm{V}}$ are, respectively, the stiffness matrix, the nodal displacement vector and the force vector, with the superscript $\mathrm{V}$ introduced to identify quantities related with the VEM domain. 
$\begin{array}{llrll}\text { October } 21, & 2021 & 12: 11 & \text { WSPC/INSTRUCTION } & \text { FILE }\end{array}$

Revised`Manuscript'UNMARKED

\subsubsection{Modeling matrix damage with Virtual Elements}

The VE formulation can be extended to problems involving nonlinear material behaviors, as detailed in Refs. 19-20. An isotropic damage model ${ }^{21,22,23,24}$ is herein adopted to model the matrix phase of the composite material. Accordingly, the loss of material integrity is caused by an equal degradation of the bulk and shear moduli and it is governed by a single internal scalar damage variable $\omega$, which tracks and measures the loss of stiffness of the material. Damage evolves monotonically within its admissible range $0 \leq \omega \leq 1$, where 0 is associated with the pristine status and 1 with the fully degraded material. The constitutive equations for an isotropic damage model are defined as

$$
\boldsymbol{\sigma}=(1-\omega) \mathbf{C}^{0} \varepsilon_{\Pi}
$$

where $\boldsymbol{\sigma}$ and $\varepsilon_{\Pi}$ collect the Voigt components of the stress and strain respectively, and $\mathbf{C}^{0}$ is the elasticity matrix for the pristine elastic material. Damage evolution is triggered upon fulfillment of the condition

$$
F(\varepsilon)=\tau(\varepsilon)-r=0, \quad r=\max _{\lambda \in \mathcal{H}}\{\tau(\lambda)\}
$$

where $\tau(\varepsilon)$ is a suitably chosen norm of the strains, used to determine if the considered stress state belongs to the elastic domain, when $F(\varepsilon)<0$, or if it induces damage initiation or evolution, $F(\varepsilon)=0$. The monotonically increasing internal variable $r$ represents the damage threshold at the current loading step $\lambda$ and it is a function of the loading history $\mathcal{H}$. To model the onset and evolution of damage in epoxy resins, often used as the matrix material in fiber-reinforced composites, Melro et al. ${ }^{25}$ proposed an expression which reads

$$
\tau(\varepsilon)=\frac{3 \tilde{J}_{2}}{X_{m}^{c} X_{m}^{t}}+\frac{\tilde{I}_{1}\left(X_{m}^{c}-X_{m}^{t}\right)}{X_{m}^{c} X_{m}^{t}},
$$

where $X_{m}^{t}$ and $X_{m}^{c}$ are, respectively, the tensile and compressive strengths of the epoxy resin. $\tilde{I}_{1}$ and $\tilde{J}_{2}$ are, respectively, the first stress invariant and the second deviatoric stress invariant, both defined using the components of the effective stress $\tilde{\boldsymbol{\sigma}}=\mathbf{C}^{0} \varepsilon_{\boldsymbol{\Pi}}$ acting in the undamaged material. The evolution of damage is governed by the Kuhn-Tucker flow rules, which read

$$
F \leq 0, \quad \dot{r} \geq 0, \quad \dot{r} F=0
$$

and allow to distinguish between loading and unloading conditions. Unloading occurs when $\dot{\tau} \leq 0$; otherwise, damage evolves and the following consistency condition must be satisfied

$$
\dot{F}=\dot{\tau}-\dot{r}=0
$$

The exponential softening damage evolution law defined as in Ref. 25 as

$$
\omega(r)=\left[1-\frac{r_{0}}{r} \exp \left(-\frac{r-r_{0}}{r_{f}-r_{0}}\right)\right] \cdot H\left(r-r_{0}\right), \quad r=\max _{\lambda \in \mathcal{H}}\{\tau(\lambda)\}
$$


is adopted to model the evolution of the damage $\omega$ after its onset. $H(\cdot)$ denotes the Heaviside step function, the parameter $r_{0}$ identifies the damage initiation condition and $r_{f}$ specifies the softening response behavior.

The nonlinear constitutive laws appearing in Eq. (11) can be treated, as in nonlinear finite element formulations, using standard incremental-iterative algorithms. The stress at a generic point $\mathbf{x}$ and at a generic loading step $\lambda$ is given by the expression

$$
\boldsymbol{\sigma}=\boldsymbol{\sigma}\left(\lambda, \mathbf{x}, \varepsilon_{\Pi}, \mathcal{H}\right)
$$

where $\varepsilon_{\Pi}$ is the approximated virtual strain computed as in Eq. (5), using the matrix projector operator $\boldsymbol{\Pi}$ and $\mathcal{H}$ contains the history variables of the damage model. The tangent material stiffness matrix $\mathbf{C}_{\text {tan }}$ at a certain time $t$ is consistently computed from the constitutive law in Eq. (17) as

$$
\mathbf{C}_{t a n}\left(t, \mathbf{x}, \varepsilon_{\Pi}, \mathcal{H}\right)=\frac{\partial \boldsymbol{\sigma}}{\partial \varepsilon_{\Pi}}
$$

\subsection{Coupling procedure for BEM and VEM subdomains}

The coupling between BEM and VEM domains is achieved according to the approach introduced in Refs. 1-2. The BEM subdomain is treated as a macro-finite element, and the traction-displacement equations associated with it are transformed into force-displacement equations and assembled with the VEM equations, already expressed in terms of nodal forces and displacements.

The vectors $\mathbf{U}^{\mathrm{V}}$ and $\mathbf{F}^{\mathrm{V}}$ appearing in Eq. (10) collect the displacement and nodal force components of all the nodes in the VEM domain. It is possible to partition the vectors as

$$
\mathbf{U}^{\mathrm{V}}=\left[\begin{array}{c}
\mathbf{U}_{S}^{\mathrm{V}} \\
\mathbf{U}_{D}^{\mathrm{V}}
\end{array}\right], \quad \mathbf{F}^{\mathrm{V}}=\left[\begin{array}{c}
\mathbf{F}_{S}^{\mathrm{V}} \\
\mathbf{F}_{D}^{\mathrm{V}}
\end{array}\right],
$$

where $\mathbf{U}_{S}^{\mathrm{V}}$ and $\mathbf{F}_{S}^{\mathrm{V}}$ identify components related to nodes belonging to the interface $S$. Along $S$, the nodal displacements and forces must satisfy the compatibility and equilibrium conditions

$$
\mathbf{U}^{\mathrm{B}}=\mathbf{U}_{S}^{\mathrm{V}}, \quad \mathbf{F}^{\mathrm{B}}+\mathbf{F}_{S}^{\mathrm{V}}=\mathbf{0},
$$

which have been written considering that no external nodal forces act on the nodes belonging to $S$. The displacement compatibility equations can be readily written, as the same displacement components appear in both the BEM and VEM equations. Contrarily, while nodal forces appear in Eq. (10), related to the VEM domain, tractions appear in Eq. (3), related to the BEM domain, so that it is necessary to retrieve consistent nodal forces from BEM tractions before writing the equilibrium equations appearing in Eq. (20). This may be done by resorting to appropriate 
$\begin{array}{lllll}\text { October } 2021 & 212: 11 & \text { WSPC/INSTRUCTION } & \text { FILE }\end{array}$

Revised'Manuscript 'UNMARKED

energetic considerations, which allow associating a system of equivalent nodal forces $\mathbf{F}^{\mathrm{B}}$ with the tractions acting over the considered boundary elements leading to

$$
\mathbf{F}^{\mathrm{B}}=\mathbf{M} \mathbf{T}^{\mathrm{B}}
$$

where $\mathbf{F}^{\mathrm{B}}, \mathbf{T}^{\mathrm{B}} \in \mathbb{R}^{2 m \times 1}$ and $\mathbf{M} \in \mathbb{R}^{2 m \times 2 m}$, being $m$ the total number of boundary nodes/elements, see Refs.1-2 for details. From Eq. (3), it follows $\mathbf{T}^{\mathrm{B}}=\mathbf{G}^{-1} \mathbf{H}^{\mathrm{B}}$, so that Eq. (21) can be rewritten as the following force-displacement equilibrium equation

$$
\mathbf{F}^{\mathrm{B}}=\mathbf{M} \mathbf{T}^{\mathrm{B}}=\left(\mathbf{M G} \mathbf{G}^{-1} \mathbf{H}\right) \mathbf{U}^{\mathrm{B}}=\mathbf{K}^{\mathrm{B}} \mathbf{U}^{\mathrm{B}} .
$$

Eq. (10), related to the VEM sub-domain, can be conveniently rewritten as

$$
\left[\begin{array}{cc}
\mathbf{K}_{S S}^{\mathrm{V}} & \mathbf{K}_{S D}^{\mathrm{V}} \\
\mathbf{K}_{D S}^{\mathrm{V}} & \mathbf{K}_{D D}^{\mathrm{V}}
\end{array}\right]\left[\begin{array}{c}
\mathbf{U}_{S}^{\mathrm{V}} \\
\mathbf{U}_{D}^{\mathrm{V}}
\end{array}\right]=\left[\begin{array}{c}
\mathbf{F}_{S}^{\mathrm{V}} \\
\mathbf{F}_{D}^{\mathrm{V}}
\end{array}\right]
$$

and it may be eventually combined with the interface conditions in Eq. (20), with Eq. (22) and with suitable external boundary conditions to obtain the problem solution.

\section{Numerical test}

In this Section, an application of the hybrid virtual-boundary element formulation, combined with an isotropic damage model for the regions modeled with virtual elements, is reported. Fig. 2 shows the model's geometry and boundary conditions: a unit cell comprising a single fiber embedded in an epoxy matrix, with initial partial debonding between fiber and matrix along the region of the interface identified by $\left|\theta_{d}\right| \leq 70^{\circ}$. Outside the debonded region, the inclusion is perfectly bonded to the matrix. The study of this fiber-matrix system has been treated extensively $27,28,29,30$. The present test aims at simulating the progression into the matrix of the two kinked cracks that start from both ends of the debonded zone. It is worth noting that, in this specific application, the coupling procedure detailed in Section 2.3 is applied only over the pristine region of the matrix-fiber interface. The debonded regions require the application of zero traction conditions over the edges of the BEM fiber domain and zero nodal equivalent forces over the nodes at the edge of the VEM matrix domain.

The side length of the unit cell is $L=0.2 \mathrm{~mm}$ and the fiber diameter is $D=$ $0.025 \mathrm{~mm}$. The center of the circle coincides with the center of the square. The tensile loading is applied by prescribing uniform displacements $\bar{u}$ at the sample left and right edges. Plane strain conditions are assumed. The fiber material is assumed linear elastic, and it does not develop damage. The matrix material is treated as linear elastic until the damage onset, governed by the loading function in Eq. (13). The exponential damage evolution law in Eq. (16) is assumed, with $r_{0}=1, r_{f}=234$, according to strength and fracture toughness data about epoxy. The transverse elastic material parameters are $E_{F}=201 \mathrm{GPa}$ and $\nu_{F}=0.22$ for 
the fiber and $E_{M}=2.8 \mathrm{GPa}$ and $\nu_{M}=0.33$ for the matrix. The fracture toughness of the epoxy matrix is $G_{f r}=0.09 \mathrm{~N} / \mathrm{mm}$.

A integral-type non local regularization approach ${ }^{31,32}$ is employed, which consists in replacing the local value of the equivalent strain $\tau\left(\mathbf{x}_{p}\right)$ at a certain point $\mathbf{x}_{p}$ with its weighted average $\bar{\tau}\left(\mathbf{x}_{p}\right)$ over a representative circular domain surrounding each material point $\mathbf{x}_{p}$

$$
\bar{\tau}\left(\mathbf{x}_{p}\right)=\int_{\Omega} \alpha\left(\mathbf{x}_{p}, \mathbf{x}_{q}\right) \tau\left(\mathbf{x}_{q}\right) d \Omega,
$$

where $\Omega$ is the analysis domain and $\alpha\left(\mathbf{x}_{p}, \mathbf{x}_{q}\right)$ is a nonlocal weight function chosen as

$$
\alpha\left(\mathbf{x}_{p}, \mathbf{x}_{q}\right)=\frac{\alpha_{0}(r)}{\int_{\Omega} \alpha_{0}(r) d \Omega},
$$

where $\alpha_{0}(r)$ is a non-negative function of the distance $r=\left\|\mathbf{x}_{p}-\mathbf{x}_{q}\right\|$ between two considered material points, monotonically decreasing for $r \geq 0$. The expression adopted for $\alpha_{0}(r)$ is the truncated quadratic polynomial function

$$
\alpha_{0}(r)=\left\langle 1-\frac{r^{2}}{R^{2}}\right\rangle^{2}
$$

where $R$ is known as the interaction radius and it is a parameter related to the characteristic length $l_{c}$. In this test, a value of $R=D / 3$ has been used.

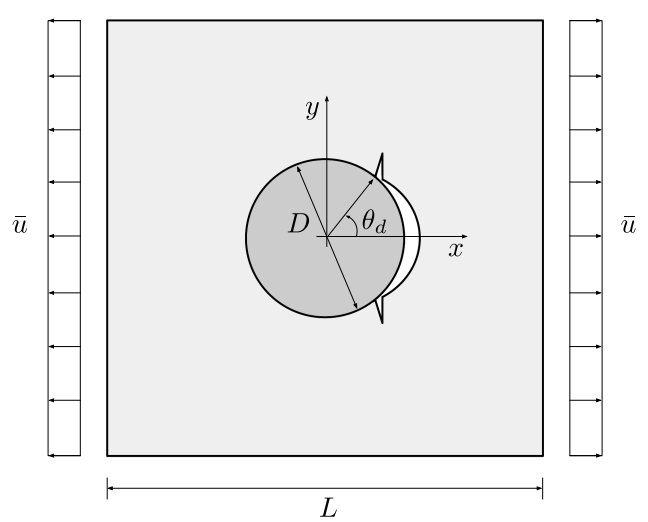

Fig. 2. Geometry and boundary conditions of the composite unit cell containing a circular fiber partially debonded from the matrix.

The matrix region is discretized with 8047 2D lowest-order virtual polygon elements, which induce 256 1D linear boundary elements on the fiber-matrix interface. The simulation is performed under displacement control using a Newton-Raphson with adaptive load step to track the steep softening branch. The simulation is arrested at a nominal macro-strain $\varepsilon_{x}=0.05$. 
Fig. 3 shows the load-displacement diagram: for each load increment, the plotted reaction force is computed as the sum of the nodal reaction forces on the right edge. The identified labels correspond to the damage profiles shown in Fig. 4. Linear elastic behavior is exhibited up to slightly before the point (a) in the curve, which marks the initiation of damage at the ends of the debonded interface, where stress concentration is expected. Once the damage is activated, the two symmetric damaged/failed region progress within the matrix, following a kinked path consistent with the previously cited references' behavior. As the load increases, the material failure evolves, affecting regions oriented perpendicularly to the load direction up to the domain boundary, which causes a progressive decrease of the load-carrying capability identified by the softening branch of the load-displacement diagram.

An important aspect related to the use of VEM for modelling evolving damage in the matrix is worth highlighting. In damage modelling with standard FEM, mesh directionality is a well-known issue, especially when highly structured meshes are employed ${ }^{33}$. In the numerical campaign, it has been noticed that the use of hexagonal elements in the VEM region, in conjunction with the use of the non-local regularisation, mitigated mesh directionality, which could be an additional indirect benefit provided by VEM. This aspect has not been however thoroughly investigated and will form the object of further investigations.

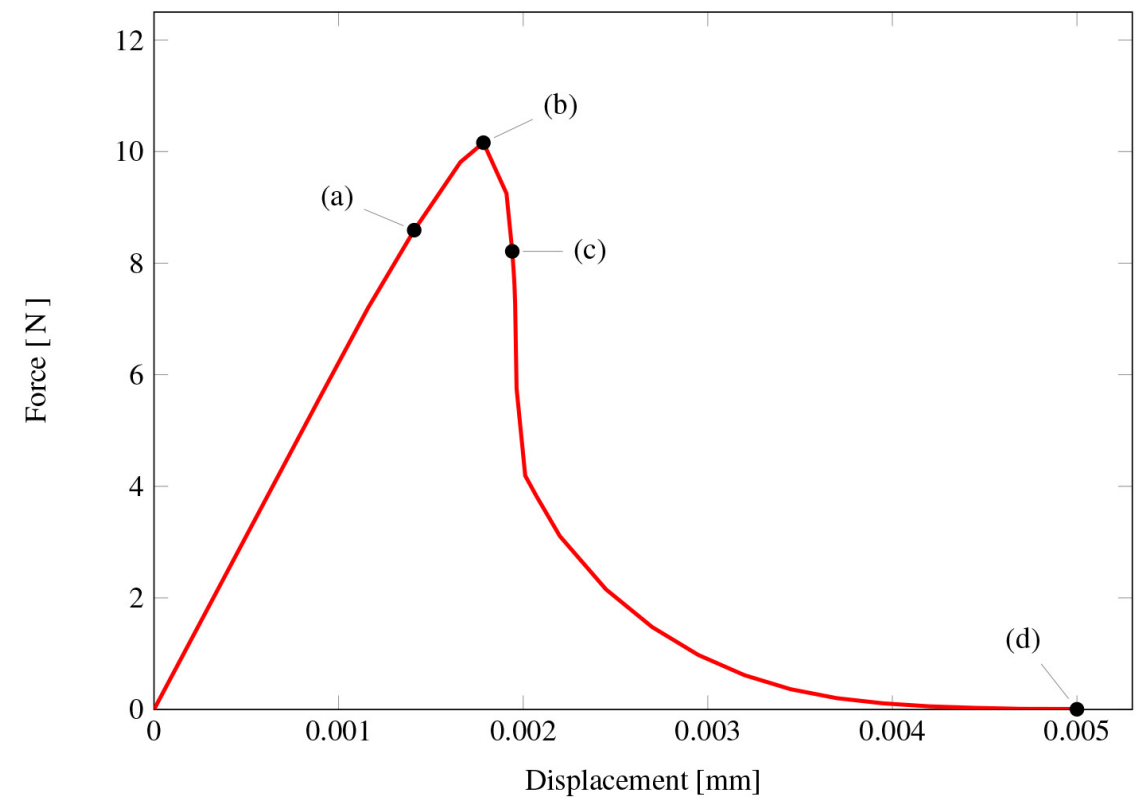

Fig. 3. Force-displacement diagram for the composite unit cell test. 


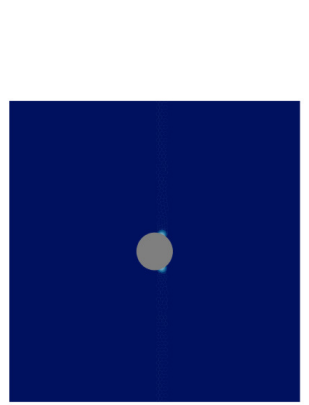

(a)

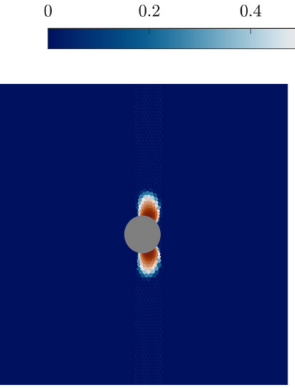

(b)

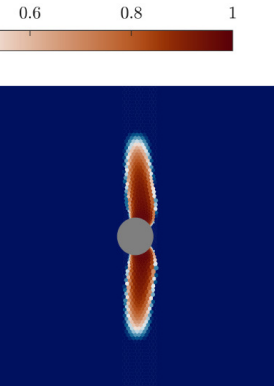

(c)

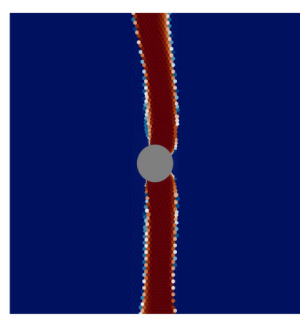

(d)

Fig. 4. Evolution of the damage profile for the composite unit cell test.

\section{Acknowledgments}

The authors acknowledge the support of the Italian Ministry of Education, University and Research MIUR through the project DEVISU, funded under the scheme PRIN-2107 Grant 22017ZX9X4K_006.

\section{References}

1. M. Lo Cascio, M. Grifo, A. Milazzo, I. Benedetti, Computational Homogenization of Heterogeneous Materials by a Novel Hybrid Numerical Scheme, Journal of Multiscale Modelling 11(04), p. 66-88 (2020).

2. M. Lo Cascio, I. Benedetti, V. Mantič, Micro damage and cracking in fibre reinforced composites by a novel hybrid numerical technique. AIP Conference Proceedings $\mathbf{2 3 0 9}$, 020001 (2020).

3. M. Lo Cascio, A. Milazzo, I. Benedetti, A hybrid virtualboundary element formulation for heterogeneous materials, Int. J. Mech. Sci. 199, 106404 (2021).

4. L. Beirão da Veiga, F. Brezzi, A. Cangiani, G. Manzini, L.D. Marini, A. Russo. Basic principles of virtual element methods, Math. Models Methods Appl. Sci. 23(01), p. 199214 (2013).

5. E. Artioli, S. Marfia, and E. Sacco, High-order virtual element method for the homogenization of long fiber nonlinear composites, Comput. Methods Appl. Mech. Eng. 341, p. $571-585(2018)$.

6. M. Lo Cascio, A. Milazzo, and I. Benedetti, Virtual element method for computational homogenization of composite and heterogeneous materials, Compos. Struct. 232, 111523 (2020).

7. M. Lo Cascio, A. Milazzo, and I. Benedetti, Virtual element method: Micro-mechanics applications. In: Key Engineering Materials 827, p. 128-133 (Trans Tech Publications Ltd), (2020).

8. P. K. Banerjee and R. Butterfield, Boundary element methods in engineering science (McGraw-Hill London, 1981).

9. M. H. Aliabadi, The boundary element method, volume 2: applications in solids and structures, Vol. 2 (John Wiley \& Sons, 2002).

10. I. Benedetti and M. Aliabadi, A three-dimensional cohesive-frictional grain-boundary 
micromechanical model for intergranular degradation and failure in polycrystalline materials, Comput. Methods Appl. Mech. Eng. 265, p. 36-62 (2013).

11. V. Gulizzi, A. Milazzo, and I. Benedetti, An enhanced grain-boundary framework for computational homogenization and micro-cracking simulations of polycrystalline materials, Comput. Mech. 56, p. 631-651 (2015).

12. I. Benedetti and M. Aliabadi, Multiscale modeling of polycrystalline materials: A boundary element approach to material degradation and fracture, Comput. Methods Appl. Mech. Eng. 289, p. 429-453 (2015).

13. V. Gulizzi and I. Benedetti, Micro-cracking of brittle polycrystalline materials with initial damage, European Journal of Computational Mechanics 25(1-2), p. 38-53, (2016)

14. I. Benedetti, V. Gulizzi, and V. Mallardo, A grain boundary formulation for crystal plasticity, Int. J. Plast. 83, p. 202-224 (2016).

15. I. Benedetti and V. Gulizzi, A grain-scale model for high-cycle fatigue degradation in polycrystalline materials, Int. J. Fatigue 116, p. 90-105 (2018).

16. I. Benedetti, V. Gulizzi, and A. Milazzo, A microstructural model for homogenisation and cracking of piezoelectric polycrystals, Comput. Methods Appl. Mech. Eng. 357, 112595 (2019).

17. F. Parrinello, V. Gulizzi, and I. Benedetti, A computational framework for low-cycle fatigue in polycrystalline materials, Comput. Methods Appl. Mech. Eng. 383, 113898 (2021).

18. E. Artioli, L. Beirão da Veiga, C. Lovadina, and E. Sacco, Arbitrary order 2D virtual elements for polygonal meshes: part I, elastic problem, Comput. Mech. 60, p. 355-377 (2017).

19. L. Beirão da Veiga, C. Lovadina, and D. Mora, A virtual element method for elastic and inelastic problems on polytope meshes, Comput. Methods Appl. Mech. Eng. 295, p. 327-346 (2015).

20. E. Artioli, L. Beirão Da Veiga, C. Lovadina, and E. Sacco, Arbitrary order 2d virtual elements for polygonal meshes: Part ii, inelastic problem, Comput. Mech. 60, p. 643-657 (2017).

21. J. Lemaitre, Local approach of fracture, Eng. Fract. Mech. 25, p. 523-537 (1986).

22. J. C. Simo and J. Ju, Strain-and stress-based continuum damage modelsi. formulation, Int. J. Solids Struct. 23, p. 821-840 (1987).

23. J. Simo and J. Ju, Strain-and stress-based continuum damage modelsii. computational aspects, Int. J. Solids Struct. 23, p. 841-869 (1987).

24. J. L. Chaboche, Continuum Damage Mechanics: Part I General Concepts, J. Appl. Mech. 55, p. 59-64 (1988),

25. A. Melro, P. Camanho, F. A. Pires, and S. Pinho, Micromechanical analysis of polymer composites reinforced by unidirectional fibres: Part iconstitutive modelling, Int. J. Solids Struct. 50, p. 1897-1905 (2013).

26. M. Jirásek, Nonlocal damage mechanics, Revue europenne de gnie civil 11, p. 9931021 (2007).

27. F. París, E. Correa, and V. Mantič, Kinking of transversal interface cracks between fiber and matrix, J. Appl. Mech. 74, p. 703-716 (2007).

28. V. Mantič, Interface crack onset at a circular cylindrical inclusion under a remote transverse tension. application of a coupled stress and energy criterion, Int. J. Solids Struct. 46, p. 1287-1304 (2009).

29. L. Távara, V. Mantič, E. Graciani, and F. París, Bem analysis of crack onset and propagation along fibermatrix interface under transverse tension using a linear elasticbrittle interface model, Eng. Anal. Bound. Elem. 35, p. 207-222 (2011).

30. I. García, M. Paggi, and V. Mantič, Fiber-size effects on the onset of fibermatrix 
$\begin{array}{lllll}\text { October } & 2021 & 12: 11 & \text { WSPC/INSTRUCTION } & \text { FILE }\end{array}$

Revised'Manuscript 'UNMARKED

debonding under transverse tension: a comparison between cohesive zone and finite fracture mechanics models, Eng. Fract. Mech. 115, p. 96-110 (2014).

31. G. Pijaudier-Cabot and Z. P. Bažant, Nonlocal damage theory, J. Eng. Mech. 113, p. $1512-1533$ (1987).

32. M. Jirásek, Nonlocal models for damage and fracture: comparison of approaches, Int. J. Solids Struct. 35(31-32), p. 4133-4145 (1998).

33. I. Benedetti, H. Nguyen, R.A. Soler-Crespo, W. Gao, L. Mao, A. Ghasemi, J. Wen, S.B. Nguyen, H.D. Espinosa, Formulation and validation of a reduced order model of $2 \mathrm{D}$ materials exhibiting a two-phase microstructure as applied to graphene oxide, J. Mech. Phys. Solids 112, p. 66-88, (2018). 